\title{
NORMES DE SYLVICULTURE POUR LES FORÉTS DE CHÊNE ROUVRE
}

\author{
J. PARDE்
}

La sylviculture des forêts de chêne (et plus spécialement des forêts de chêne rouvre du secteur ligérien) est un peu une spécialité françaişe dont on reconnaît du reste la valeur hors de nos frontières. Aussi bien le sujet est-il souvent abordé dans cette revue. Citons par exemple Lorne en 1961 - clôturant alors un "dialogue " ouvert en 1956 - Venet en 1967, Bourgenot en 1970 .

Nous-mêmes, à partir des places d'expérience de la Station de sylviculture et de production du Centre national de recherches forestières (C.N.R.F.), avons construit en 1962 une table de production qui a été reprise dans le fascicule des "Tables de production pour les forêts françaises", présentées par l'Institut national de la recherche agronomique (I.N.R.A.) et I'Ecole nationale du génie rural, des eaux et des forêts en 1973 sous la conduite de Décourt ( ${ }^{1}$ ).

Bouchon donnait en 1970 une "Norme provisoire pour le chêne de qualité du secteur ligérien", et Venet, dans le cadre de l'enseignement et de la formation continue à l'Ecole nationale du génie rural, des eaux et des forêts, a rédigé de nombreux documents stencilés de grande valeur - les plus récents pour la session de 1977 "Sylviculture du chêne" - que connaissent bien les ingénieurs forestiers français.

Laissant de côté la phase difficile et mal connue de la régénération naturelle - assistée ou non - notre propos est maintenant d'informer nos lecteurs des travaux poursuivis à la Station de sylviculture et de production de l'Institut national de la recherche agronomique pour mettre au point des normes pouvant servir de guides à la culture des peuplements réguliers de chêne. Nous donnons ici au mot "norme" la même définition que Bourgenot (1970) : il s'agit d'une loi. empirique ou non, qui rend compte de la progression souhaitable des nombres de tiges au fur et à mesure qu'un peuplement vieillit. On la traduit sous forme d'un tableau - ou d'une courbe - qui indique ce que doivent être les nombres des tiges jugés optima devant exister dans un peuplement à tous les âges de sa vie, entre sa naissance et la récolte (compte tenu, ajouteronsnous, du type de sylviculture choisi préalablement $\left({ }^{2}\right)$.

La norme qu'on pouvait déduire de notre table de production était une norme moyenne basée sur les données chiffrées récoltées de 1925 à 1960 dans 31 places d'expérience d'éclaircie du chêne suivies en forêts de Bellême (Orne), Blois (Loir-et-Cher), et Tronçais (Allier). II s'agissait

1) Ce fascicule, rassemblant dix tables de production, peut toujours être commandé au Service des Publications du Centre de Nancy de l'Ecole du génie rural, des eaux el des forèts, 14, rue Girardet, 54042 NANGY CEDEX.

2) Pour chaque forêt, plusieurs sylvicultures sont bien sûr possibles, L'amenagiste, en fixant tel objectif de production, telle priorité économique, emporte en fait la décision. Nous n'en discuterons pas ici : au praticien de choisir, dans chaque cas particulier la norme qui lui paraît convenir au mieux á son problème particulier. 


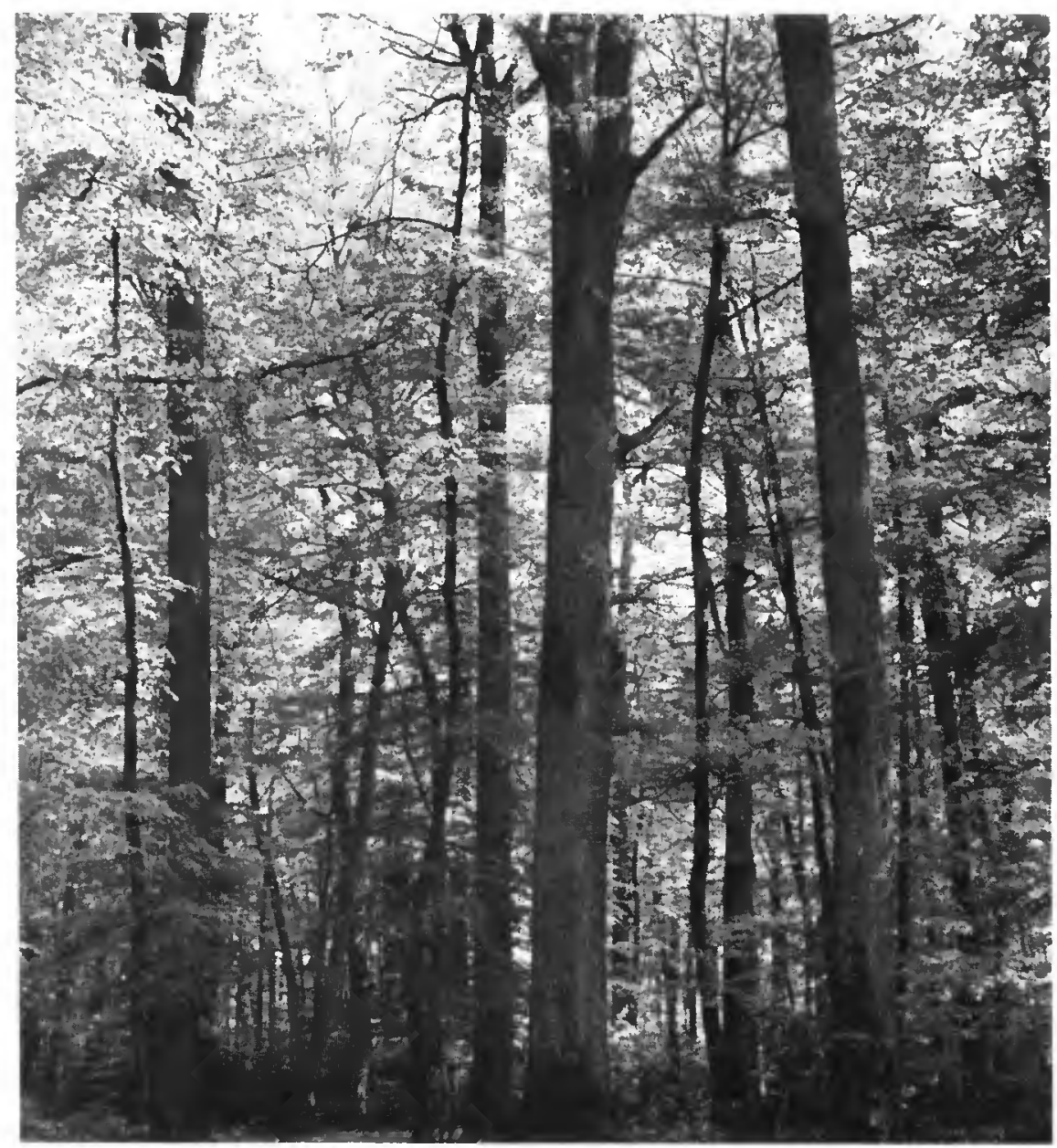

Futaie de chêne rouvre.

Photo TOULGOUAT.

d'une sylviculture classique dans la première moitiè du siècle, mise en œuvre par nos prédécesseurs : disons quelque 1000 tiges sur pied à l'ha à 50 ans, 350 à 100 ans, $100 / 120$ à 200 ans.

On sait maintenant que la sylviculture du chêne peut être plus hardie sans baisse de produc. tion, et Bouchon (1970) après avoir travaillé dans quatre groupes de forêts : Orne, Sarthe, Loir-et-Cher, Allier, a pu donner la progression suivante, permettant de maintenir constamment des accroissements annuels de $2 \mathrm{~mm}$ sur le rayon : 1000 tiges à 50 ans, 210 tiges à 100 ans, 72 tiges à 200 ans.

En fait, rendre compte de la variation du nombre des tiges en fonction des âges présente de sérieux inconvénients : ne serait-ce que celui de nécessiter la présentation d'une progression différente chaque fois que varie le niveau de fertilité des stations. D'où l'idée de définir les nombres de tiges en fonction des hauteurs dominantes (hauteur moyenne des 100 plus gros arbres à l'hectare). On obtient ainsi une "loi" beaucoup plus générale, indépendante des niveaux locaux de fertilité : il suffira, chaque fois, de mesurer la hauteur dominante réelle, pour pouvoir se référer aux tableaux ou graphiques existant. La "loi " en question peut, en 
suffisante approximation, être relativement simple : en utitisant par exemple les données récoltées sur les places d'expérience pour la table de production Pardé (1962) on calcule une droite fort bien ajustée au nuage de points en portant :

- en abscisse, graduation normale, les hauteurs dominantes;

- en ordonnée, graduation logarithmique, les nombres de tiges successifs après éclaircies.

C'est cette représentation graphique souvent vérifiee, assez inattendue peut-on avouer, que nous employons maintenant couramment à la Station de sylviculture et de production, et que nous utiliserons dans la suite de cette étude, qui propose d'admettre les principes simplificateurs suivants, très acceptables lorsqu'il s'agit de transférer de façon simple au praticien des données scientifiques plus complexes:

Deux principes de base

- La production forestière totale d'un peuplement pur équienne est une fonction directe de la hauteur dominante, quel que soit le milieu: les hauteurs successives étant atteintes d'autant plus vite que la station est plus fertile (Delvaux, 1975).

- La sylviculture d'un peuplement pur équienne peut se définir par une norme précisant les nombres de tiges successifs à maintenir sur pied en fonction de la progression des hauteurs dominantes du peuplement. Cette norme peut être valablement représentée par une droite si l'on porte en abscisse les hauteurs dominantes, en ordonnée tes logarithmes des nombres de tiges.

\section{Deux principes complèmentaires}

- La nature des éclaircies est "programmèe" par l'adoption d'un facteur (Décourt, 1972). II s'agit du rapport $K=\frac{V m}{V g}$ du volume de l'arbre moyen $V m$ enlevé en éclaircie à celui de l'arbre moyen du peuplement sur pied avant éclaircie ( $\left.{ }^{3}\right)$.

- La rotation des éclaircies peut excellement s'apprécier en fonction d'un certain accroissement de la hauteur dominante: on décidera par exemple, dans le cas du chêne et en sylviculture intensive de passer en éclaircie chaque fois que la hauteur dominante aura crû de un mètre : en peuplement de tertilité moyenne, cela donne un passage tous les six ans environ avant 80 ans, tous les huit ans de 80 à 120 ans, tous les dix ans de 120 à 150 ans, tous les douze à quinze ans ensuite.

Ceci ètant, les éclaircies ont continue depuis 1960 dans les places d'expérience de la Station de sylviculture et de production, en secteur ligérien comme en Lorraine. Les mesures dendromètriques correspondantes ont suivi, d'autres expérimentations ont été lancées, notamment par Bouchion et Oswald, de nouveaux résultats ont été acquis. Le moment est venu de faire le point, et de conforter un dossier où trop d'interrogations subsistent encore.

Pour y parvenir de façon claire, nous présenterons l'affaire sous forme d'un tableau, puis d'un graphique (dû pour l'essentiel à Oswald) que nous commenterons successivement.

\section{Le tableau}

Il indique volontairement de façon sommaire - ce qui ne l'empêche pas de pouvoir servir de guide - cinq types de sylviculture "chêne " que nous suivons - ou tentons de suivre - dans les places d'experience de la Station de sylviculture et de production. II rend compte des nom.

3) Pour une essence donnee, Decourt se lixe du reste $K$ en tonction de la hauteur et du nombre de tiges $N$, el méme en fonc. tion de $N$ seutement, avec un excellent ajustement.

Ottorını (1975) a étudié la valıdıte de ce coeffıcıent $K$, el a confırmé pour le chène qu'on pouvait l'exprimer, quelle que soıt l'essence en cause, par une courbe d'équalion. $K=\operatorname{Exp}(a \log N+b N+c)$. 
LA SYLVICULTURE DU CHÊNE :

Nombre de tiges en fonction des âges

\begin{tabular}{|c|c|c|c|c|c|c|c|c|c|}
\hline Modalitè sylvicole & 40 ans & 60 ans & $\begin{array}{c}70 \text { ans } \\
\text { Hdam } 18 / 22 \mathrm{~m}\end{array}$ & 80 ans & 90 ans & $\begin{array}{c}100 \text { ans } \\
\text { Hdom } 23 / 26 \mathrm{~m}\end{array}$ & 110 ans & 130 ans & $\begin{array}{c}200-230 \text { ans } \\
(\text { Hdom }=35 \mathrm{~m})\end{array}$ \\
\hline $\begin{array}{l}\text { 1. Lorraine et secteur ligé- } \\
\text { rien - recherche - sans } \\
\text { eclaircies autres que } \\
\text { martalité naturelle (ma- } \\
\text { ximum biologiquement } \\
\text { possible) .......... } \\
\text { 2. Lorraine - recherche - } \\
\text { éclaircies faibles ..... } \\
\text { 3. Secteur ligérien - } \\
\text { recherche "classique" } \\
\text { 4. Lorraine et secteur ligé- } \\
\text { rien - recherche - éclair- } \\
\text { cies modérées puis fortes } \\
\text { 5. Eclaircies très fortes - } \\
\text { recherche - "norme } 85 \text { " }\end{array}$ & $\begin{array}{l}3400 \\
2200 \\
1600\end{array}$ & $\begin{array}{r}1700 \\
1200 \\
730\end{array}$ & $\begin{array}{l}700 \\
580\end{array}$ & 1100 & $\begin{array}{l}500 \\
350\end{array}$ & 510 & $\begin{array}{l}420 \\
310\end{array}$ & $\begin{array}{c}450 \\
? \\
220\end{array}$ & $\begin{array}{c}? \\
\text { objectif : } 140 \text { à } 150 \\
110\end{array}$ \\
\hline
\end{tabular}

bres de tiges en fonction des âges (mais des indications sont données sur les hauteurs) et se rapproche dans toute la mesure du possible des données réelles existant dans nos dossiers.

Il faut le lire dans l'ordre que voici :

Modalité 2 - Lorraine - éclaircies faibles.

II s'agit d'un type d'éclaircies faibles mis en route il y a 50 ans dans plusieurs places d'expérience d'âges échelonnés, et que nous continuons à mener sans rien changer au protocole initial. L'objectif est de terminer avec quelque 150 tiges sur pied à 200 ans.

Modalité 3 - secteur ligérien - recherche "classique".

C'est la sylviculture de notre table de production, qui reflète la situation de tout un groupe de places d'experiences en secteur ligérien. Cette sylviculture correspond à celle qui peut être suivie sans difficulté dans beaucoup de peuplements existants. Nous rappelons qu'elle nécessite tout de même de faire partir en éclaircies $54 \%$ de la production totale en volume bois fort tige de l'origine à 200 ans (48\% à 150 ans). Elle a l'inconvénient de maintenir sur pied un nombre final de tiges jugé maintenant trop élevé pour des raisons bien connues (il est possible de faire grossir plus vite les arbres en les desserrant davantage, sans perdre le bénéfice de la qualitè tranchage).

Modalité 4 - Lorraine et secteur ligérien - recherche - éclaircies modérées, puis fortes.

II s'agit d'une rectification en cours d'expérience dans une partie de nos places "éclaircies modérées ", de la sylviculture antérieure, afin d'intensifier les éclaircies programmèes et aboutir à un nombre final de tiges de 100 seulement. On y parvient souvent, et obtient ainsi une variante de la table de production "Pardé 1962 ".

Modalité 5 - éclaircies très fortes - recherche - norme 85.

Notre collaborateur $\mathrm{H}$. Oswald tente d'ajuster et suivre dans certaines places d'expériences menées jusqu'à maintenant avec des éclaircies déjà assez fortes une "sylviculture normalisée " terminant en fin de révolution avec quelque 85 tiges seulement à l'hectare, sans entre temps perturber exagérément le peuplement. C'est là "manœuvre " difficile, et cela d'autant plus que la rectification de tir a lieu dans un peuplement plus âgè. 
Ces difficultés apportent la preuve qu'un changement de cap en sylviculture ne se décrète pas simplement sur papier : I'histoire passèe du peuplement en cause est très contraignante, et tout n'est pas possible : il est illusoire de penser mener sans à-coup, à disons 80 tiges seulement en fin de révolution, des peuplements de chêne d'âge moyen, prévus auparavant pour terminer leur existence avec 120 tiges environ à l'hectare. Et c'est là un cas très fréquent.

Modalité 1 "sans éclaircies autres que la mortalité naturelle".

On se borne à enlever tous les 5 ans les arbres morts, ou sur le point de mourir. Le nombre des tiges vivantes sur pied est donc le maximum biologiquement tolèrable à l'âge correspondant, compte tenu des particularités de la Station.

Il s'agit là de "placeaux-témoins ", dont le plus ancien du genre est suivi depuis 1885 !

\section{Le graphique}

Si donc il est intéressant de savoir qu'il est difficile de conduire à moins de 100 tiges à l'hectare des peuplements d'âge moyen maintenus trop denses pendant la première moitié de leur révolution, s'il est possible de terminer avec $85 / 90$ tiges à l'hectare des peuplements semblables mais plus jeunes, il vaut plus généralement la peine de savoir de quelle "plage de manœuvre" dispose le sylviculteur aux différents âges pour faire varier la sylviculture sans risquer de perdre une part notable de production ligneuse qui, dans le cas du chêne, r'est déjá pas tellement importante $\left(^{4}\right)\left(5\right.$ à $6 \mathrm{~m}^{3}$ au mieux de bois fort tige d'accroissement moyen annuel à 150/200 ans, dont la moitiè "part" en éclaircies à des prix souvent peu avantageux). Ce que nous venons de préciser pour la modalitè précédente (pas d'éclaircies, si ce n'est l'auto-éclaircie, la "self-tninning" des anglo-saxons) fournit déjà une frontière véritablement infranchissable : il est sans doute biologiquement impossible de maintenir sur pied des peuplements de chêne plus denses.

A l'opposé, les travaux de Bouchon visent à étudier ce que doit être un nombre minimum de tiges maintenues sur pied dans la suite des âges pour que :

- les cimes des arbres ne se gênent jamais et se bornent à se "tangenter " avant le pas. sage des éclaircies,

- les accroissements sur le diamètre soient constamment réguliers : $1,5 \mathrm{~mm}$ ou $2 \mathrm{~mm}$ ou $2,5 \mathrm{~mm}$,

- la production ne chute pas significativement de sa valeur stationnelle normale.

Bouchon dispose ainsi de trois normes:

- une norme chêne, accroissements moyens annuels sur le diamètre 1,5 mm, 130 tiges à I'hectare à 200 ans,

- une norme chêne, accroissements moyens annuels $2 \mathrm{~mm}, 72$ tiges à l'hectare à 200 ans,

- une norme chêne, accroissements moyens annuels $2,5 \mathrm{~mm}, 55$ tiges à l'hectare à 200 ans.

II note : "la norme correspondant aux accroissements de $2,5 \mathrm{~mm}$ suppose de très fortes éclaircies. Il est remarquable qu'à àge égal les volumes finaux, dans ce cas, soient très inférieurs à ceux des deux autres normes qui eux, par contre, sont sensiblement equivalents ". Cela veut dire que la frontière, en-dessous de laquelle chutent fortement les productions ligneuses potentielles, se situe entre les normes " 70 tiges à 200 ans" et " 55 tiges à 200 ans " (nous dirons, pour simplifier, $N 70$ et $N 55$ ) et sans doute assez près de la norme $N 70$ que nous pourrons considérer comme limite inférieure (et celà d'autant plus volontiers qu'au-delà la qualité tranchage ne'semble plus assurée...).

4) Disons á ce sujet que, lorsqu'un peuplement de chẽne èvolue sans éclaircies, la mortalité naturelle - non récoltée - fait disparaître peu à peu une proportion de la production ligneuse qui n'est pas négligeable: 20 a $25 \%$ au tolal á un âge de $110 / 120$ ans, d'aprés nos places d'expériences (maximum observé : plus de $30 \%$ à 120 ans). 


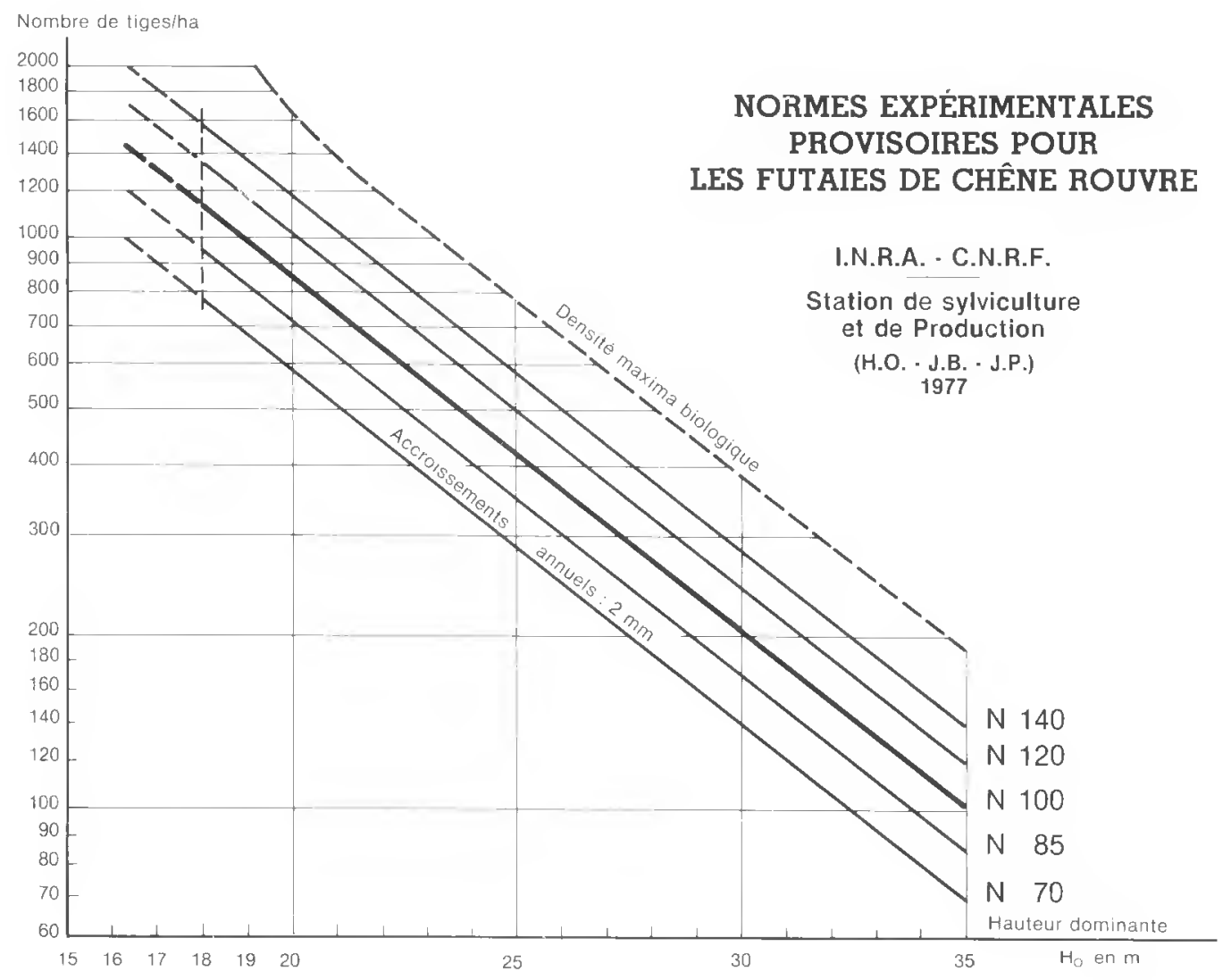

Pour y voir plus clair, utilisons la représentation graphique dont nous venons de parler.

Les diftérentes sylvicultures en question se traduisent chacune par une droite à coefficient angulaire négatit. Et ces droites sont pratiquement parallèles les unes aux autres: la pratique torestière peut en tout cas se contenter d'un schéma ainsi simplifié.

Les deux courbes encadrantes:

- norme N 70 (il s'agira encore d'une droite);

- densité maxima biologiquement tolérable (qu'on ne peut, elle, compte tenu de nos données, "réduire " à une droite de bout en bout);

délimitent, à peu de choses près, une plage dans laquelle le sylviculteur peut "manœuvrer" sans porter atteinte à la quantité de bois produite.

Cette "liberté " va même jusqu'à la possibilité de passer, en cours de révolution, d'une courbe à sa voisine immédiate : mais bien sûr, cette facilité diminue au tur et à mesure que le peuplement prend de l'âge. Dans un perchis de $14 / 15 \mathrm{~m}$ de hauteur dominante (40 à 50 ans), tout est encore possible. Jusqu'à 90 ans (environ 23 mètres de hauteur dominante), nous arrivons, difficultueusement toutetois, à la station de Sylviculture, à taire passer telle place d'expérience d'un régime $-\mathrm{N} 100$ par exemple - au régime immédiatement inférieur $-\mathrm{N} 85$.

Passé 100 ans, les jeux sont fait, définitivement, les modèles sylvicoles sont figés, et leur remise en cause peut être nocive. 
On remarquera que les normes s'arrêtent, côté de l'axe des ordonnées, à la hauteur dọminante 18 mètres.

C'est qu'effectivement, plus à gauche (donc pour des peuplements plus jeunes), les données de nos dossiers ou bien sont absentes, ou bien sont contradictoires. C'est aussi que les "densités initiales" définies fort bien par Bartet comme "le nombre de tiges vivantes au moment où l'on envisage la première éclaircie du peuplement" sont très variábles : 3000 tiges par exemple à $14 \mathrm{~m}$ de hauteur dominante et 36 ans dans une place d'expérience issue de plantation près de Nancy, 6000 au même âge à Tronçais dans une place d'expérience issue de régénération naturelle.

Mais il peut arriver d'avoir à commencer les éclaircies dans des perchis ayant bien moins de 3000 tiges à l'ha - 1500 seulement par exemple - à l'opposé, la place d'expérience de Tronçais que nous évoquons avait 14600 tiges sur pied dix ans plus tôt, à 25 ans d'âge. On a même noté, en forêt domaniale de Blois, 17500 tiges à 30 ans dans une régénération naturelle!

Or cette densité initiale a une importance capitale pour la sylviculture qui suivra (Bartet, 1976). Passer en quelque 20 ou 30 ans de 1500 tiges à moins de 1000 pose sans doute moins de problèmes que de passer, dans le même laps de temps, de 15000 à 1000 . Quelle est la rapidité d'évolution des situations existantes à ce moment-là ? Quelle "mobilité " potentielle individuelle subsiste encore, comment jouent alors les phénomènes de concurrence?

Il est vite trop tard en sylviculture.

Comment faire bien évoluer sans les perturber gaulis et perchis ? Beau sujet d'étude à engager pour un organisme de recherche sylvicole et qui ne concerne pas que le chêne!

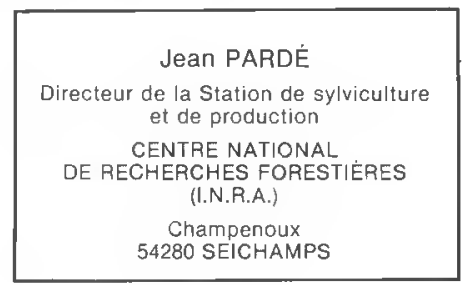

\section{BIBLIOGRAPHIE}

BARTET (J.H.), BOLLIET (R.). - Méthode utilisée pour la construction de tables de production à sylviculture variable. Bulletin de l'Office national des forêts, section technique. Document $n^{\circ} 76-9,1976$.

BOUCHON (J.). - Norme provisoire pour le chêne de qualité du secteur ligérien. - Nancy-Champenoux, Centre national de recherches forestières - Station de sylviculture et de production, 1970. - 13 p. (document à diffusion restreinte).

BOURGENOT (L.). - Le traitement des tutaies teuillues productrices de bois d'œuvre de qualité. Revue forestière française, $\mathrm{n}^{\circ} 1,1970$, pp. 25-33.

DECOURT (N.). - Méthode utilisée pour la construction rapide de tables de production provisoires en France. Annales des sciences forestiéres, vol. 1, 1972, pp. 35-48.

DECOURT (N.). - Tables de production pour les forêts françaises. - Nancy, Ecole nationale du génie rural, des eaux et des forêts, 1973.

DELVAUX $\left(J_{1}\right)$. - Les éclaircies numériques. - $94^{\mathrm{e}}$ congrès de l'Association française pour l'avancement des sciences. Bruxelles, 1975 (communication 17-04).

LORNE (A.). - La sylviculture de l'avenir (toujours à propos du chêne de qualité). Revue forestière française, $n^{\circ} 7,1961$, pp. 503-514.

OTTORINI (J.M.). - En marge du calcul des tables de production pour le Pin noir dans le sud-est de la France. - Nancy-Champenoux, Centre national de recherches forestières - Station de sylviculture et de produc tion, 1975 (document à diffusion restreinte).

VENET (J.M.). - Sylviculture des forêts de chêne de tranchage. Revue forestière française, $n^{\circ} 12,1967$, pp. 746-758. 